PROCEEDINGS OF THE

AMERICAN MATHEMATICAL SOCIETY

Volume 132, Number 1, Pages 273-282

S 0002-9939(03)06987-9

Article electronically published on April 24, 2003

\title{
ON BERRY-ESSEEN BOUNDS OF SUMMABILITY TRANSFORMS
}

\author{
J. A. FRIDY, R. A. GOONATILAKE, AND M. K. KHAN
}

(Communicated by Claudia M. Neuhauser)

\begin{abstract}
Let $Y_{n, k}, k=0,1,2, \cdots, n \geq 1$, be a collection of random variables, where for each $n, Y_{n, k}, k=0,1,2, \cdots$, are independent. Let $A=\left[p_{n, k}\right]$ be a regular summability method. We provide some rates of convergence (Berry-Esseen type bounds) for the weak convergence of summability transform $(A Y)$. We show that when $A=\left[p_{n, k}\right]$ is the classical Cesáro summability method, the rate of convergence of the resulting central limit theorem is best possible among all regular triangular summability methods with rows adding up to one. We further provide some summability results concerning $\ell^{2}$-negligibility. An application of these results characterizes the rate of convergence of Schnabl operators while approximating Lipschitz continuous functions.
\end{abstract}

\section{INTRODUCTION}

Let $Y:=\left[Y_{k, m}\right]$ be a matrix of random variables, where $\left(Y_{k, m}, k=0,1,2 \cdots\right)$ is the $m$-th column vector consisting of mutually independent random variables with finite variances, $m=0,1,2, \cdots$. Let $A:=\left[p_{n, k}\right]$ be a summability matrix. Consider

$$
S_{n}:=\sum_{k=0}^{\infty} \frac{p_{n, k}\left(Y_{n, k}-E\left(Y_{n, k}\right)\right)}{\left\|\sigma_{n} p_{n}\right\|_{2}}=: \sum_{k=0}^{\infty} X_{n, k},
$$

where

$$
X_{n, k}:=\frac{p_{n, k}\left(Y_{n, k}-E\left(Y_{n, k}\right)\right)}{\left\|\sigma_{n} p_{n}\right\|_{2}}, \quad\left\|\sigma_{n} p_{n}\right\|_{2}^{2}:=\sum_{k=0}^{\infty} p_{n, k}^{2} \operatorname{Var}\left(Y_{n, k}\right)<\infty .
$$

We will present some results concerning the weak convergence of the sequence $S_{n}$, $n \geq 1$. Since different summability methods have different convergence fields, one expects to see the dependence of rates of convergence of $S_{n}$ on the choice of $A$. Consequently, a natural question is to ask if there is a summability transform that leads to the fastest rate of convergence. We will show that the classical Cesáro transform provides the fastest rate among all regular triangular methods whose row sums equal one.

Convergence, in probability and almost sure sense, of such transforms have already been settled by various authors $([3,4,4,[13,[16])$. In this paper we will show

Received by the editors August 3, 2001 and, in revised form, August 22, 2002.

2000 Mathematics Subject Classification. Primary 60F05; Secondary 41A36, $40 \mathrm{C} 05$.

Key words and phrases. Approximation operators, central limit theorem, convolution methods, Schnabl operators. 
that through summability theory we may unify the weak convergence part with the classical central limit theorem and the classical Berry-Esseen (BE) bound. The Cesáro method gives the classical result and the Abel method, for instance, gives the discounted central limit theorem of actuarial sciences ([10], 2] ). The interesting part is that the tools remain the same as found in the standard books of probability (see [5], 8]).

The next section collects some summability results that are relevant for our later developments, and perhaps may have some independent interest in summability theory. Then we present results concerning the optimal rate of BE bounds among triangular summability methods. The last section provides a link to approximation theory.

\section{Some SUMmaBility RESUlts}

Let $A=\left[a_{n, k}\right], n, k \geq 0$, be an infinite matrix of complex numbers. We say that a sequence $x:=\left(x_{0}, x_{1}, \cdots\right)$ is in the domain of $A$ if the series

$$
(A x)_{n}:=\sum_{k=0}^{\infty} x_{k} a_{n, k}
$$

is convergent for each $n=0,1,2, \cdots$. When $x$ is in the domain of $A$, the transformed sequence $(A x)_{n}, n=0,1,2, \cdots$, is denoted by $(A x)$. If $c$ denotes the set of all convergent complex sequences, then $c_{A}:=A^{-1}(c)$ denotes the set of all sequences $x$ such that $(A x)$ is convergent. We say that $B$ includes $A$ if $c_{A} \subseteq c_{B}$. If it obeys the strict inclusion, i.e., if $c_{A} \subset c_{B}$, then $B$ is said to be stronger than $A$. Let $I$ denote the identity matrix. We say $A$ is regular if $c_{I} \subseteq c_{A}([1])$.

One of the most well-known regular summability methods is the Cesáro method, denoted by $(C, 1)$, in which $a_{n, k}=\frac{1}{n+1}$ for $k=0,1, \cdots, n$, and zero otherwise. If we define $a_{n, k}=\left(\begin{array}{l}n \\ k\end{array}\right) r^{k}(1-r)^{n-k}$ for $0 \leq k \leq n$ and $n \geq 1, a_{0,0}=1$, we get the Euler method, $E_{r}$, which is regular if and only if $r \in(0,1]$ (cf. [15]). A large class of regular summability methods can be obtained by using two nonnegative integer valued random variables as follows.

Definition 2.1. Let $U$ and $V$ be two nonnegative integer valued random variables. Let $V_{1}, V_{2}, \cdots$ be mutually independent random variables identically distributed as $V$. Let $\left[p_{n, k}\right]$ be a matrix whose $n$-th row consists of the discrete probability density of the random variable $U+V_{1}+V_{2}+\cdots+V_{n}$. We will call $\left[p_{n, k}\right]$ the convolution method generated by $U$ and $V$.

It is easy to see that the Euler method $E_{r}$ is a convolution method. When we take $U$ and $V$ as Poisson(1) random variables, the resulting convolution method is known as the Borel matrix method. By using $U$ and $V$ as geometric, or shifted geometric random variables, one gets the Taylor and Meyer-König summability methods. By using the Silverman-Teoplitz theorem one can easily show that the convolution method is regular if and only if $P(V=0)<1$.

Definition 2.2. We say $A=\left[a_{n, k}\right]$ is $\ell^{2}$-negligible if

$$
\left\|a_{n}\right\|_{2}^{2}:=\sum_{k=0}^{\infty}\left|a_{n, k}\right|^{2} \rightarrow 0, \quad \text { as } n \rightarrow \infty \text {. }
$$


The $\ell^{2}$-negligibility concept is closely tied to the asymptotic negligibility concept that applies in the central limit theorem. It happens to be the case that most of the standard regular summability methods are $\ell^{2}$-negligible. For instance, the Euler method is $\ell^{2}$-negligible as long as the parameter $r \in(0,1)$. We will show that the regular convolution methods (different from the identity matrix) are $\ell^{2}$-negligible. Further, we will show that any method which includes an $\ell^{2}$-negligible regular summability method is also $\ell^{2}$-negligible. The following proposition contains a result which, for the most part, is well known in probability literature (see for instance [5]). The last part is a relevant addition to it, using the Pringsheim double limit concept.

Proposition 2.1. Let $T=\left[t_{n, k}\right]$ be a complex matrix. Then

$$
\lim _{n \rightarrow \infty} \sum_{k}\left|t_{n, k}\right|^{2}=0 \Rightarrow \lim _{n \rightarrow \infty} \max _{k}\left|t_{n, k}\right|=0 .
$$

Also, if $T$ satisfies the condition

$$
\sum_{k}\left|t_{n, k}\right| \leq M<\infty, \text { for all } n \geq 0
$$

then the following are equivalent:

(1) $\lim _{n \rightarrow \infty} \max _{k}\left|t_{n, k}\right|=0$.

(2) $T$ is $\ell^{2}$-negligible.

Furthermore, if $\left[t_{n, k}\right]$ satisfies the uniform boundedness condition (2.1) and its columns tend to zero, then the above two statements are equivalent to

(3) $\lim _{n, k \rightarrow \infty}\left|t_{n, k}\right|=0$,

where $\lim _{n, k}$ represents the Pringsheim double limit in the sense that for any $\epsilon>0$ there exists an $N$ so that $\left|t_{n, k}\right|<\epsilon$ for both $n, k>N$.

Proof. Since $\max _{k}\left|t_{n, k}\right|^{2} \leq \sum_{k}\left|t_{n, k}\right|^{2} \rightarrow 0$, the first part follows trivially. To show the converse after assuming (2.1), note that

$$
\sum_{k}\left|t_{n, k}\right|^{2} \leq\left(\max _{k}\left|t_{n, k}\right|\right) \sum_{k}\left|t_{n, k}\right| \leq\left(\max _{k}\left|t_{n, k}\right|\right) M \rightarrow 0 .
$$

Now to prove that (3) implies (1), let $\epsilon>0$. Then by (3), there exists an $N_{1}$ such that $\left|t_{n, k}\right|<\epsilon$ for all $k, n \geq N_{1}$. Since the columns tend to zero, we can make the tail entries of the first $N_{1}$ columns less than $\epsilon$ for large $n$. That is, there is an $N_{2}$ such that $\left|t_{n, k}\right|<\epsilon$ for $0 \leq k<N_{1}$ for all $n \geq N_{2}$. Thus, $\max _{k}\left|t_{n, k}\right|<\epsilon$ for all $n \geq \max \left\{N_{1}, N_{2}\right\}$. To prove the converse, assume that (2) is true but (3) is false. Then, there exists an $\epsilon>0$ and infinitely many $n_{j}$ and $k_{j}=k\left(n_{j}\right)$ such that both are increasing to infinity and

$$
\left|t_{n_{j}, k_{j}}\right|>\epsilon, \quad j=1,2, \cdots .
$$

But then

$$
\sum_{k}\left|t_{n_{j}, k}\right|^{2} \geq\left|t_{n_{j}, k_{j}}\right|^{2}>\epsilon^{2}
$$

for infinitely many $j$ contradicting (2).

The following proposition is well known in probability literature when the matrix is row finite $([5],[9])$. The proof remains similar; therefore, it is omitted. 
Proposition 2.2. Let $\left[t_{n, k}\right]$ be a complex matrix which satisfies the following conditions:

(1) $\sum_{k}\left|t_{n, k}\right| \leq M<\infty$, for all $n \geq 0$,

(2) $\sum_{k} t_{n, k} \rightarrow 1$ as $n \rightarrow \infty$ and

(3) the matrix $\left[t_{n, k}\right]$ is $\ell^{2}$-negligible.

Then for any complex number $z$ we have

$$
\lim _{n \rightarrow \infty} \prod_{k=1}^{\infty}\left(1+t_{n, k} z\right)=e^{z}
$$

The following result shows that for two regular methods $A$ and $B$, if $B$ includes $A$ and $A$ is $\ell^{2}$-negligible, then so is $B$.

Theorem 2.3. If $A=\left[a_{n, k}\right]$ and $B=\left[b_{n, k}\right]$ are regular matrices such that

$$
\lim _{n, k} a_{n, k}=0 \quad \text { and } \quad \lim _{n, k} b_{n, k} \neq 0,
$$

then $B$ cannot include A.

Proof. We will use the classical sliding-hump argument for the proof. First note that since the rows of $A$ are null sequences, the fact that $\lim _{n, k} a_{n, k}=0$, we have

$$
\lim _{k \rightarrow \infty} \max _{n}\left|a_{n, k}\right|=0
$$

Also, since $\lim _{n, k} b_{n, k} \neq 0$, we can choose increasing sequences of row and column indices satisfying

$$
\left|b_{\nu(m), \kappa(m)}\right| \geq \delta>0, \quad \text { for all } m .
$$

Then use (2.2) to choose a subsequence of these pairs $(\nu(m), \kappa(m))$ such that

$$
\max _{n}\left|a_{n, \kappa(m)}\right|<2^{-m} \text {. }
$$

Next, the fact that the columns of $A$ and $B$ are null sequences, we choose a further subsequence so that for all $m$ (i.e., for all $\kappa(m)), \nu(m)$ is chosen so that $k<\kappa(m)$, $n>\nu(m)$ implies

$$
\left|a_{n k}\right|<2^{-m} \quad \text { and } \quad\left|b_{n k}\right|<2^{-m} \text {. }
$$

Use the fact that the rows of $B$ tend to zero in order to get $\left|b_{n k}\right|<2^{-m}$ for all $k>\kappa(m)$ and $n<\nu(m)$. Define the sequence $x$ by

$$
x_{k}:=\left\{\begin{array}{cl}
m+1 & \text { if } k=\kappa(m), \text { for } m=0,1, \cdots, \\
0 & \text { if } k \neq \kappa(m), \text { for } m=0,1, \cdots .
\end{array}\right.
$$

This yields (for $n>\nu(m)$ )

$$
\begin{aligned}
\left|(A x)_{n}\right| & =\left|\sum_{j=0}^{\infty} a_{n, \kappa(j)}(j+1)\right| \\
& \leq\left|\sum_{j \leq m} 2^{-m}(j+1)\right|+\left|\sum_{j>m} 2^{-j}(j+1)\right| \\
& =2^{-m} \frac{(m+1)(m+2)}{2}+R_{m},
\end{aligned}
$$


where $R_{m} \rightarrow 0$. Therefore, $(A x)$ converges to zero. Also,

$$
\begin{aligned}
(B x)_{\nu(m)} & =\sum_{j=0}^{\infty} b_{\nu(m), \kappa(j)} x_{k(j)} \\
& =b_{\nu(m), \kappa(m)}(m+1)+\sum_{j \neq m} b_{\nu(m), \kappa(j)}(j+1) \\
& =b_{\nu(m), \kappa(m)}(m+1)+\sum_{j \leq m} 2^{-m}(j+1)+\sum_{j>m} 2^{-j}(j+1) \\
& =b_{\nu(m), \kappa(m)}(m+1)+2^{-m-1} m(m+1)+R_{m} .
\end{aligned}
$$

Since the first term in (2.4) is unbounded and the latter two terms tend to zero, it follows that $(B x)$ is not even bounded and hence could not be convergent. This shows that $B$ cannot include $A$.

Remark 2.4. It is not necessary to assume the full strength of regularity to have the proof work. It would be sufficient to assume (2.2) instead of assuming that $\lim _{n, k} a_{n, k}=0$ and that $A$ and $B$ have null columns and rows. Or, it would be sufficient to assume the existence of $\{\kappa(m)\}$ such that $\lim _{k} \max _{m}\left|a_{n, \kappa(m)}\right|=0$, and for a corresponding $\{\nu(m)\}$ we have $\left|b_{\nu(m), \kappa(m)}\right| \not \rightarrow 0$.

\section{BE BOUNDS FOR SUMMABILITY TRANSFORMS}

In this section we present the summability analog of the BE bound and show that the Cesáro transform provides, in some sense, the best rate of convergence. By using Propositions 2.1 and 2.2 and the standard results of probability theory one can verify the following version of Lyapunov's theorem.

Theorem 3.1. Let $S_{n}=\sum_{k=0}^{\infty} X_{n, k}$ be a random series, where $X_{n, k}, k=0,1,2 \cdots$, are independent. Further, assume that $E\left|X_{n, k}\right|^{3}=\gamma_{n, k}<\infty, E\left(X_{n, k}\right)=0$, and $\sum_{k=0}^{\infty} \operatorname{Var}\left(X_{n, k}\right)=1$. If

$$
\Gamma_{n}:=\left\|\gamma_{n}^{1 / 3}\right\|_{3}^{3}=\sum_{k=0}^{\infty} \gamma_{n, k} \rightarrow 0, \quad \text { as } n \rightarrow \infty,
$$

then $S_{n}$ converge in distribution to the standard normal random variable.

We now present Berry-Esseen type bounds for $S_{n}$. The results are derived from the generalization of the classical Berry-Esseen theorem ([5], [4]) to particular summability methods such as the Abel method and the Zeta method ([6], [10], and [14]). The approach of this proof uses the technique of characteristic functions that is already available in the literature (4] and [5]), however, with an appropriate modification to incorporate infinitely many terms in the sums. We will omit the details. The central limit theorem for summability methods considered in [7] and [12] deal primarily with non-uniform rates of convergence.

Theorem 3.2. Let $S_{n}=\sum_{k=0}^{\infty} X_{n, k}$ be a random series, where $X_{n, k}, k=0,1,2, \cdots$, are independent. Further, assume that $E\left|X_{n, k}\right|^{3}=\gamma_{n, k}<\infty, E\left(X_{n, k}\right)=0$, and $\sum_{k=0}^{\infty} \operatorname{Var}\left(X_{n, k}\right)=1$. Then

$$
\left|P\left(S_{n} \leq x\right)-\frac{1}{\sqrt{2 \pi}} \int_{-\infty}^{x} e^{-u^{2} / 2} d u\right| \leq C \Gamma_{n},
$$

where $C$ is an absolute constant and $\Gamma_{n}:=\left\|\gamma_{n}^{1 / 3}\right\|_{3}^{3}=\sum_{k=0}^{\infty} E\left(\left|X_{n, k}\right|^{3}\right)$. 
We will now examine the bound, $\Gamma_{n}$, when

$$
X_{n, k}:=\frac{p_{n, k} Y_{k}}{\left\|\sigma p_{n}\right\|_{2}}, \quad E\left(Y_{k}\right)=0, \quad\left\|\sigma p_{n}\right\|_{2}^{2}:=\sigma^{2} \sum_{k=0}^{\infty} p_{n, k}^{2},
$$

and $Y_{0}, Y_{1}, \cdots$ are iid random variables with $\operatorname{Var}\left(Y_{0}\right)=\sigma^{2}$. The aim is to discover which summability transform gives the best rate. The following theorem shows that the class of convolution summability methods cannot be the one that gives the best rate.

Theorem 3.3. Let $\left[p_{n, k}\right]$ be the convolution summability method generated by $U$ and $V$. If $U$ and $V$ have finite variances, then, $\Gamma_{n}=O\left(n^{-1 / 4}\right)$ as $n \rightarrow \infty$. Furthermore, this rate cannot be improved.

Proof. Since

$$
\left\|p_{n}\right\|_{3} \leq\left(\max _{k}\left|p_{n, k}\right| \sum_{k}\left|p_{n, k}\right|^{2}\right)^{1 / 3}
$$

we have $\left\|p_{n}\right\|_{3} \leq\left\|p_{n}\right\|_{\infty}^{1 / 3}\left\|p_{n}\right\|_{2}^{2 / 3}$. This gives that

$$
\frac{\left\|p_{n}\right\|_{3}}{\left\|p_{n}\right\|_{2}} \leq \frac{\left\|p_{n}\right\|_{\infty}^{1 / 3}\left\|p_{n}\right\|_{2}^{2 / 3}}{\left\|p_{n}\right\|_{2}}=\frac{\left\|p_{n}\right\|_{\infty}^{1 / 3}}{\left\|p_{n}\right\|_{2}^{1 / 3}} .
$$

Let $S_{n}=U+V_{1}+V_{2}+\cdots+V_{n}$ and let $S_{n}^{\prime}$ be independent and identically distributed as $S_{n}$. Note that

$$
\sum_{k} p_{n, k}^{2}=\sum_{k} P\left(S_{n}=k\right) P\left(S_{n}^{\prime}=k\right)=P\left(S_{n}=S_{n}^{\prime}\right) .
$$

Let $Z_{i}:=V_{i}-V_{i}^{\prime}, i=1,2, \cdots, n$, where $V_{i}^{\prime}$ are independent and identically distributed as $V_{i}$. If $\operatorname{Var}\left(V_{1}\right)=\sigma^{2}$, we have (see, for instance, [5])

$$
n^{1 / 2} P\left(\sum_{k=1}^{n} Z_{k}=j\right) \rightarrow \frac{1}{2 \sigma \sqrt{\pi}}, \quad p_{n, k}=P\left(S_{n}=k\right) \leq \frac{C}{\sqrt{n}}
$$

for any integer $j$, for all $k$ and some absolute constant $C$. Hence,

$$
\begin{aligned}
n^{1 / 2} P\left(S_{n}-S_{n}^{\prime}=0\right) & =n^{1 / 2} \sum_{j=-\infty}^{\infty} q_{j} P\left(\sum_{k=1}^{n} Z_{k}=j\right) \\
& \rightarrow \sum_{j=-\infty}^{\infty} q_{j} \frac{1}{2 \sigma \sqrt{\pi}},
\end{aligned}
$$

where $\left\{q_{j}\right\}$ is the probability density of $U-U^{\prime}$. Therefore, $\left[p_{n, k}\right]$ is $\ell^{2}$-negligible, and

We have

$$
n^{1 / 4}\left\|p_{n}\right\|_{2} \rightarrow \frac{1}{\sqrt{2 \sigma} \pi^{1 / 4}}
$$

$$
\frac{\left\|p_{n}\right\|_{3}}{\left\|p_{n}\right\|_{2}} \leq \frac{\left\|p_{n}\right\|_{\infty}^{1 / 3}}{\left\|p_{n}\right\|_{2}^{1 / 3}} \leq C n^{-1 / 12}
$$

for some constant $C>0$. This gives the required assertion. 
To prove that the rate is best possible, take $\left[p_{n, k}\right]$ to be the Euler method. If $|k-n r| / \sqrt{n r(1-r)}<K$, then there exists a constant $D>0$ (which depends only on $r$ and $K$ ) such that

$$
\left|\left(\begin{array}{l}
n \\
k
\end{array}\right) r^{k}(1-r)^{n-k}-\frac{1}{\sqrt{2 \pi r(1-r) n}} \exp \left\{-(k-n r)^{2} /(2 n r(1-r))\right\}\right| \leq \frac{D}{n} .
$$

Thus, if $A_{n, K}:=\{k:|k-n r| / \sqrt{n r(1-r)}<K\}$ is the set of all non-negative integers $k$ for which the stated inequality holds, we have

$$
\begin{aligned}
\sum_{k=0}^{\infty} p_{n, k}^{3} & \geq \sum_{k \in A_{n, K}} p_{n, k}^{3} \\
& \geq \min _{k \in A_{n, K}} p_{n, k}^{2} \sum_{k \in A_{n, K}} p_{n, k} \\
& \geq\left\{\frac{e^{-2 K^{2}}}{(2 \pi r(1-r) n)^{2 / 2}}+O\left(\frac{1}{n^{1.5}}\right)\right\} \sum_{k \in A_{n, K}} p_{n, k} .
\end{aligned}
$$

By the central limit theorem,

$$
\sum_{k \in A_{n, K}} p_{n, k}=P\left(\left(S_{n}-n r\right) / \sqrt{n r(1-r)}<K\right) \rightarrow P(|Z|<K),
$$

where $Z$ is the standard normal random variable. Thus, for any $K>0$, we have

$$
\liminf _{n} n \sum_{k=0}^{\infty} p_{n, k}^{3} \geq\left\{\frac{e^{-2 K^{2}}}{(2 \pi r(1-r))}\right\} P(|Z|<K)>0 .
$$

That is, there exists a $\delta>0$ such that

$$
\liminf _{n} n^{1 / 3}\left\|p_{n}\right\|_{3} \geq \delta>0 .
$$

Since

we see that

$$
\lim _{n} n^{1 / 4}\left\|p_{n}\right\|_{2}=\frac{1}{\pi^{1 / 4} \sqrt{2 r(1-r)}}
$$

$$
\liminf _{n} n^{1 / 12} \frac{\left\|p_{n}\right\|_{3}}{\left\|p_{n}\right\|_{2}}=\liminf _{n} \frac{n^{1 / 3}\left\|p_{n}\right\|_{3}}{n^{1 / 4}\left\|p_{n}\right\|_{2}} \geq C>0 .
$$

This proves the theorem.

In the statistical literature the concept of a minimum variance unbiased estimator is used to identify a best possible estimator (among the class of all unbiased estimators). Since the variance usually goes to zero as the sample size gets large, one only compares the variances of competing unbiased estimators that rely upon the same sample size. In a similar context, the comparison of rates of convergence in the central limit theorem will make sense if the competing summability methods use the same "sample size". Hence, if we identify $X_{0}, X_{1}, X_{2}, \cdots, X_{n-1}$ as our sample of size $n$, then the $n$-th terms of the competing transforms could be compared if the summability methods are forced to be triangular. In the following theorem we therefore assume that the summability methods are triangular.

The Silverman-Toeplitz theorem states that if the method is regular, then its row sums (even though they may not equal one for any row) must, in the limit, 
become one. To avoid the "contamination" in the BE rate that is caused by the row sums not being equal to one, but converging to one, we assume that each row of the summability method adds up to one.

Theorem 3.4. For the case of iid sequences of random variables, over the class of all triangular real regular summability methods (with rows adding up to one) the rate of convergence, $\Gamma_{n}$, in the Berry-Esseen bound has the fastest rate of decrease when we use the Cesáro summability method.

Proof. Let $r, s>0$ so that $\frac{1}{r}+\frac{1}{s}=1$. For any numbers $a_{k}$

$$
\begin{aligned}
\sum_{k=1}^{n}\left|a_{k}\right|^{p} & =\sum_{k=1}^{n}\left|a_{k}\right|^{p} \cdot 1 \\
& \leq\left\{\sum_{k=1}^{n}\left|a_{k}\right|^{p r}\right\}^{p /(p r)} \cdot n^{1 / s}=\|a\|_{p r}^{p} \cdot n^{1 / s} .
\end{aligned}
$$

This gives that $\|a\|_{p}^{p} \leq\|a\|_{p r}^{p} \cdot n^{\frac{r-1}{r}}$ or $\|a\|_{p} \leq\|a\|_{p r} \cdot n^{\frac{r-1}{p r}}$. The equality holds if and only if the vector $a$ and the constant vector 1 are parallel. For $p=2$, and $r=\frac{3}{2}$ we get

$$
\|a\|_{2} \leq\|a\|_{3} \cdot n^{\frac{1}{6}}
$$

In the Hölder inequality the equality takes place if and only if the vectors are parallel. That is, the vector $a$ is a constant vector. Since the rows have to add up to one, the constant vector must be $(1 / n, 1 / n, \cdots, 1 / n)$.

\section{Schnabl approximation operators}

Let $X_{0}, X_{1}, \cdots$ be a sequence of independent and identically distributed random variables taking values in an interval $I$. We will assume that the third moment exists and denote $E\left(X_{0}\right)=x$. Let $A=\left[a_{n k}\right]$ be a regular summability method with rows adding up to one. The Schnabl approximation operator, 1], is defined by

$$
L_{n}(f, x):=E f\left((A X)_{n}\right), \quad n=1,2, \cdots .
$$

Usually $A$ is assumed to be triangular. Let $\omega$ be a modulus of continuity defined over $[0, \infty)$ i.e., $\omega(t)$ is a non-decreasing continuous function with $\omega\left(0^{+}\right)=\omega(0)=0$ and $\omega(t+s) \leq \omega(t)+\omega(s)$. The space $H^{\omega}(I)$ consists of those functions $f: I \rightarrow \Re$ so that $|f(u)-f(v)| \leq \omega(|u-v|)$, and is called the Lipschitz space generated by $\omega$. The following result quantifies the rate of convergence of Schnabl operators.

Theorem 4.1. For the above Schnabl operators, we have

(1) For any $f \in H^{\omega}(I),\left|L_{n}(f, x)-f(x)\right|=O\left(\omega\left(\left\|a_{n}\right\|_{2}\right)\right)$.

(2) If $\operatorname{Var}\left(X_{0}\right)>0$ and $\left\|a_{n}\right\|_{3}=o\left(\left\|a_{n}\right\|_{2}\right)$, then the rate cannot be improved in (1).

Proof. We will use the fact that if $\omega$ is a modulus of continuity, then there exists a concave modulus of continuity $\omega^{*}$ so that $\omega \leq \omega^{*} \leq 2 \omega$. When $f \in H^{\omega}(I)$, we have

$$
\begin{aligned}
\left|L_{n}(f, x)-f(x)\right| & \leq E\left|f\left((A X)_{n}\right)-f(x)\right| \\
& \leq E \omega\left(\left|(A X)_{n}-x\right|\right) \\
& \leq 2 \omega\left(E\left|(A X)_{n}-x\right|\right) \\
& \leq 2 \omega\left(\left\|a_{n}\right\|_{2}\right)\left(1+E \frac{\left|(A X)_{n}-x\right|}{\left\|a_{n}\right\|_{2}}\right) .
\end{aligned}
$$


The last expression remains bounded. To prove the reverse inequality, take $f(t)=$ $\omega(|t-x|)$ which belongs to $H^{\omega}(I)$. Since

$$
|f(t)| \leq(|t|+1) \omega(1)+\omega(|x|)
$$

we see that $L_{n}(f, x)$ is well defined. Next let $Z_{n, x}:=\left((A X)_{n}-x\right) /\left\|a_{n}\right\|_{2}$. Note that

$$
\begin{aligned}
\left|L_{n}(f, x)-f(x)\right| & =E \omega\left(\left\|a_{n}\right\|_{2}\left|Z_{n, x}\right|\right) \\
& \geq \omega\left(\left\|a_{n}\right\|_{2}\right) E\left(\frac{\left|Z_{n, x}\right|}{1+\left|Z_{n, x}\right|}\right) \\
& \geq c \omega\left(\left\|a_{n}\right\|_{2}\right),
\end{aligned}
$$

for a $c>0$ and all large $n$, since when $\left\|a_{n}\right\|_{3}=o\left(\left\|a_{n}\right\|_{2}\right)$,

$$
E\left(\frac{\left|Z_{n, x}\right|}{1+\left|Z_{n, x}\right|}\right) \rightarrow E\left(\frac{|Z|}{1+|Z|}\right)>0, \quad Z \sim N(0,1)
$$

Among the regular triangular methods that have row sums one, Theorem 4.1 suggests that the best rate of convergence for the Schnabl operators is achieved if we use the Cesaro summability method. This class contains the classical approximation operators such as Bernstein, Szasz, Baskakov, Gamma and Weierstrass operators. For each one of them, the rate is $\omega\left(n^{-1 / 2}\right)$ ([1]). On the other hand, when $A$ is taken to be the Euler method, the resulting Schnabl operators inherit the rate $\omega\left(n^{-1 / 4}\right)$.

\section{REFERENCES}

1. F. Altomare and M. Campiti, Korovkin type Approximation Theory and its Applications, Walter de Gryter Publ. Berlin, 1994. MR 95g:41001

2. N. L. Bowers; H. U. Gerber; J. C. Hickman; D. A. Jones, and C. J. Nesbitt, Actuarial Mathematics, Second edition, The Society of Actuaries, Schaumburg, Illinois, 1999.

3. Y. S. Chow, Delayed sums and Borel summability of independent, identically distributed random variables, Bull. Inst. Math. Acad. Sinica. 1 (1973), no. 2, 207-220. MR 49:8099

4. Y. S. Chow and H. Teicher, Probability theory, independence, interchangeability, martingales, Second edition, Springer Verlag, 1988. MR 89e:60001

5. K. L. Chung, A course in probability theory, Harcourt, Brace \& World, Inc., New York, 1974. MR 49:11579

6. P. Diaconis, Weak and strong averages in probability theory and the theory of numbers, $\mathrm{Ph} . \mathrm{D}$. thesis, Dept of Statistics, Harvard University, May 1974.

7. P. Embrechts, and M. Maejima, The central limit theorem for summability methods of i.i.d. random variables, Z. Wahrsc. Verw. Gebiete. 68 (1984), no. 2, 191-204. MR 86f:60038

8. W. Feller, An introduction to probability theory and it's applications, Vol. II, Second edition, John Wiley and Sons, New York, 1966. MR 35:1048

9. B. R Gelbaum, Some theorems in probability theory, Pacific J. Math. 118 (1985), no 2, 383391. MR 86i:60042

10. H. U. Gerber, The discounted central limit theorem and its Berry-Esseen analogs, Ann. Math. Statist. 42 (1971), 389-392. MR 43:1255

11. G. H. Hardy, Divergent series, Clarendon Press, Oxford, 1949. MR 11:25a

12. Y. Kasahara, and M. Maejima, Functional limit theorems for weighted sums of i.i.d. random variables, Probab. Theory Relat. Fields. 72 (1986), no. 2, 161-183. MR 88b:60086

13. T. L. Lai, Summability methods for independent, identically distributed random variables, Proc. Amer. Math. Soc. 45 (1974), 253-261. MR 50:8665

14. E. Omey, A limit theorem for discounted sums, Z. Wahrsch. Verw. Gebiete. 68 (1984), no. 1, 49-51. MR 86c:60034 
15. R. L. Powell, and S. M. Shah, Summability theory and applications, Van Nostrand Reinhold, London, 1972

16. W. E. Pruitt, Summability of independent random variables, J. Math. Mech., 15 (1966), no. 5, 769-776. MR 33:3338

Department of Mathematical Sciences, Kent State University, Kent, Ohio 44242

E-mail address: fridy@math.kent.edu

Department of Mathematics, Texas A\&M International University, Laredo, Texas 78041

E-mail address: harag@tamiu.edu

Department of Mathematical Sciences, Kent State University, Kent, Ohio 44242

E-mail address: kazim@math.kent.edu 